ISSN 0103-5150

Fisioter. Mov., Curitiba, v. 26, n. 1, p. 193-202, jan./mar. 2013

Licenciado sob uma Licença Creative Commons

\title{
Exercícios físicos e osteoartrose: uma revisão sistemática
}

\author{
Exercise and osteoarthrosis: a systematic review
}

\section{Vanderlane de Souza Duarte ${ }^{[a]}$, Marcelo Lasmar dos Santos ${ }^{[b]}$, Kleicy de Abreu Rodrigues ${ }^{[c]}$, John Barreto Ramires ${ }^{[\mathrm{d}]}$, Guilherme Peixoto Tinoco Arêas ${ }^{[\mathrm{e}]}$, Grasiely Faccin Borges ${ }^{[\mathrm{f}]}$}

[a] Fisioterapeuta pelo Instituto de Saúde e Biotecnologia da Universidade Federal do Amazonas (Ufam), Coari, AM - Brasil, e-mail: vanderlaneduarte@msn.com

[b] Acadêmico do curso de Medicina da Universidade do Estado do Amazonas, Manaus (UEA), AM - Brasil, e-mail: marcelo_lasmar@hotmail.com

[c] Fisioterapeuta pelo Instituto de Saúde e Biotecnologia da Universidade Federal do Amazonas (Ufam), Coari, AM - Brasil, e-mail: kleicy_abreu@hotmail.com

[d] Fisioterapeuta pelo Instituto de Saúde e Biotecnologia da Universidade Federal do Amazonas (Ufam), Coari, AM - Brasil, e-mail: johndiegocoari@hotmail.com

[e] Mestre em Ciências Fisiológicas pela Universidade Federal do Espírito Santo (Ufes), professor do Curso de Fisioterapia do Instituto de Saúde e Biotecnologia da Universidade Federal do Amazonas, Coari, AM - Brasil, e-mail: guilhermepta@hotmail.com

[f] Bolsista de doutorado Pleno no Exterior (CNPq) da Faculdade de Ciências do Desporto e Educação Física da Universidade de Coimbra, Coimbra-Portugal, professora assistente do Instituto de Saúde e Biotecnologia da Universidade Federal do Amazonas (Ufam), Coari - AM- Brasil, e-mail: gfborges@ufam.edu.br

\section{Resumo}

Introdução: A osteoartrose (OA) é uma doença articular crônico-degenerativa que se evidencia pelo desgaste da cartilagem articular e que atinge frequentemente as articulações que sustentam peso, como o joelho. Objetivo: Relatar as principais evidências associadas ao uso do exercício físico na melhora dos sintomas decorrentes da osteoartrose em indivíduos acima de 50 anos. Materiais e métodos: Trata-se de uma revisão sistemática de literatura, com a utilização das bases de dados eletrônicos de periódicos indexados: National Library of Medicine (MEDLINE - PubMed), Literatura Latino-Americana e do Caribe em Ciência da Saúde (LILACS), e a base Scientific Electronic Library Online (SciELO). Resultados: Foram encontrados 1.405 artigos, selecionados nas bases de dados SciELO, LILACS, PubMed e MEDLINE; destes, 101 artigos foram selecionados. Entre as regiões acometidas pela osteoartrose, o joelho foi o local mais mencionado, seguido do joelho 
e quadril juntos; outras regiões foram citadas com menor recorrência. Os exercícios que tiveram maior destaque foram exercícios aquáticos, isocinéticos, treinamento de força muscular, caminhada, além da educação em saúde que também foi muito utilizada nos estudos selecionados. Conclusão: Pôde-se constatar por esta revisão que os estudos demonstram a eficácia do uso de exercícios na melhora dos sintomas decorrentes da osteoartrose, porém, não há um consenso quanto aos parâmetros de aplicação, como intensidade e duração de cada tipo de exercício.

Palavras-chave: Osteoartrite. Exercício físico. Terapêutica. Fisioterapia.

\section{Abstract}

Introduction: Osteoarthritis $(\mathrm{OA})$ is a chronic degenerative joint disease that is evidenced by the wear of joint's cartilage, in which often reaches the joints that support weight, like knee. Objective: To describe the principal evidence associated with the use of exercise in improving symptoms of osteoarthritis resulting in individuals over 50 years. Materials and methods: This is a systematic review of literature with the use of electronic databases of journals indexed: National Library of Medicine (MEDLINE - PubMed), Latin American and Caribbean Center on Health Sciences (LILACS), and based Scientific Electronic Library Online (SciELO). Results: There were 1405 articles were selected in the databases ScIELO, LILACS, PUbMed and MEDLINE with these 101 articles were selected. Among the areas affected by osteoarthritis, the knee was the most frequently mentioned, followed by knee and hip together, other regions were also cited with less intensity. The exercises that have become more prominent were water exercises, isokinetic, muscle strength training, walking, and health education has also been widely used in selected studies. Conclusion: It was found that through this review that studies show the efficacy of exercise in improving the symptoms caused by osteoarthritis, but there is no consensus on the application parameters such as intensity and duration of each exercise.

Keywords: Osteoarthritis. Physical exercise. Therapeutics. Physical therapy.

\section{Introdução}

A osteoartrose $(\mathrm{OA})$ é uma doença articular crônico-degenerativa que se evidencia pelo desgaste da cartilagem articular (1). Clinicamente, a osteoartrose caracteriza-se por dor, rigidez matinal, crepitação óssea, atrofia muscular e quanto aos aspectos radiológicos é observado estreitamento do espaço intra-articular, formações de osteófitos, esclerose do osso subcondral e formações císticas $(1,2)$.

É uma afecção bastante comum e se apresenta entre $44 \%$ e $70 \%$ dos indivíduos acima de 50 anos de idade; na faixa etária acima de 75 anos, esse número eleva-se a $85 \%$. Além disso, representa uma das principais queixas da consulta médica e é responsável por um número exorbitante de absenteísmo e aposentadorias por invalidez (2).

0 período mais comum de início do desenvolvimento da afecção é entre 50 e 60 anos (3). Estima-se que entre a população acima de 65 anos, aproximadamente $12 \%$ tendem a ter osteoartrose sintomática, que é caracterizada principalmente por relato doloroso principalmente em períodos matinais em uma ou mais articulações do corpo $(1,3)$. A incidência da osteoartrose sintomática aumenta com a idade e com o peso corporal. As articulações do joelho, as interfalângicas distais, carpometacárpicas e as articulações facetarias são as mais afetadas (1).

As alterações mais significativas ocorrem nas superfícies articulares que perdem a sua congruência (4). Enquanto isso acontece na cartilagem, o osso subcondral sofre alterações proliferativas. Essas alterações ocorrem na margem das articulações e no assoalho das lesões cartilaginosas, que por sua vez comprometem a elasticidade e aumentam a rigidez óssea, tornando os ossos mais sensíveis ao desenvolvimento de microfraturas $(1,3)$. Essas microfraturas regeneram-se, porém, de forma excessiva; isso ocasiona a formação de calos ósseos e, consequentemente, aumenta a rigidez que compromete toda a estrutura articular dando origem aos osteófitos, luxações e instabilidade articular. Proliferação sinovial e sinovite ativa também são comuns (4). As células 
da sinovia perto da periferia tornam-se metaplásicas e acarretam a formação de novos osteófitos (5).

Quanto às alterações metabólicas, sabe-se que as taxas de síntese e de secreção de enzimas que degradam a matriz e as células da cartilagem articular (3). A atividade enzimática lisossômica e extralisossômica apresentam-se elevadas quando comparadas com valores referenciais de normalidade. Essas enzimas levam à degradação dos agregados e à perda de proteoglicanas da matriz óssea, e colágeno tipo II (6). 0 arcabouço proteico das proteoglicanas causam a ativação de outras enzimas, entre elas a colagenase, que por sua vez destrói o colágeno, o qual é, aparentemente, o fator principal na progressão da patologia e na destruição final da superfície articular $(3,6)$.

Basicamente, existem dois tipos de osteoartrose; considerando a etiologia, esta pode ser classificada como primária, quando não existe uma causa conhecida, ou como secundária, quando é desencadeada por fatores conhecidos e determinados (1). Na osteoartrose, primária ou secundária, a cartilagem é o tecido com maiores alterações (2). Entre as alterações morfológicas, a cartilagem articular perde sua natureza homogênea e é rompida e fragmentada, com fibrilação, fissuras e ulcerações (1). Às vezes, com o avanço da patologia, não resta nenhuma cartilagem e áreas de osso subcondral ficam expostas $(2,3)$.

Os exercícios físicos atualmente vêm sendo utilizados tanto no tratamento quanto na prevenção de diversas patologias crônico-degenerativas entre elas a osteoartrose (2). Terapia física local, reabilitação, exercícios, redução dos fatores mecânicos sobre a articulação e ainda terapias alternativas podem proporcionar à pessoa acometida melhorias no quadro álgico e manutenção da função articular, o que reflete principalmente no ganho de qualidade de vida dessas pessoas. A fisioterapia também está entre uma das formas de terapia física de tratamento para a osteoartrose e, quando bem indicada, oferece excelentes respostas (4).

As principais situações que indicam o emprego de exercícios físicos no tratamento da osteoartrose são: dor e rigidez articular, perda da mobilidade articular sem destruição importante da articulação, desalinhamento articular ou uso anormal da articulação, sintomas de fraqueza muscular, fadiga e resistência cardiovascular reduzida e ainda alterações da marcha e do equilíbrio $(1,4)$.

Um profissional bem treinado e com conhecimento sobre as atuais alternativas de tratamento é indispensável para que bons resultados sejam alcançados e para que orientações sejam feitas de maneira específica e individualizada para cada paciente $(1,4)$. Desse modo, conhecer a patologia e seu tratamento a partir dos exercícios físicos é indispensável para que o profissional de saúde aplique e elabore uma melhor conduta, com o intuito de proporcionar à pessoa acometida uma melhoria, principalmente no que se refere ao quadro sintomatológico e, consequentemente, proporcionar melhorias na sua qualidade de vida.

O objetivo do presente trabalho foi relatar as principais evidências associadas ao uso do exercício físico/terapêutico na melhora dos sintomas decorrentes da osteoartrose em indivíduos acima de 50 anos através de uma revisão sistemática de literatura.

\section{Materiais e métodos}

A presente investigação foi sustentada através de revisão sistemática de literatura, com a utilização das bases de dados eletrônicos de periódicos indexados; foram analisadas as publicações a partir do ano 2000 até o ano de 2008, na base da National Library of Medicine, na base de dados PubMed, na base da Literatura Latino-Americana e do Caribe em Ciência da Saúde (LILACS), e na base Scientific Electronic Library Online (SciELO).

Esta investigação envolveu a busca por estudos que possuíram como descritores os termos osteoartrose, artrose, osteoartrite e exercícios, na língua portuguesa; e osteoarthritis, osteoarthrosis e exercise, na língua inglesa, a fim de verificar a relação entre esses estudos.

Para a seleção dos artigos científicos, foram estabelecidos os seguintes critérios: apresentar informações no estudo sobre indivíduos acometidos pela osteoartrose, com idade superior a 50 anos de idade, relatar informações sobre a utilização de exercícios físicos e/ou terapêuticos no tratamento da doença.

Os procedimentos foram organizados na seguinte sequência: na primeira etapa da investigação, realizou-se um levantamento de artigos encontrados com os descritores propostos nas bases de dados anteriormente mencionadas; em uma segunda etapa, ocorreu uma leitura e seleção criteriosa dos artigos e a formação de um banco de dados sistematizado. Nessa etapa, os dados de todos os artigos incluídos foram coletados e armazenados em novo banco de dados, além das características sociodemográficas da 
população estudada, país, ano, tipo de estudo, formas de coletas de dados, autores, entre outras variáveis que se mostraram interessantes para a investigação. Os artigos foram agrupados por similaridade de população e resultados, de forma que se caracterizou a publicação científica que se associasse à temática.

Na etapa de análise, esses dados foram debatidos conforme a literatura pertinente, no sentido de estabelecer consensos e pontos divergentes na literatura e produzir um resumo crítico sintetizando as informações disponibilizadas pelos artigos coletados.

A terceira etapa contou com a sistematização e análise dos artigos dos bancos de dados formados.

Foram critérios de exclusão: 1) artigos em outros idiomas que não português e/ou inglês; 2) trabalhos científicos que estivessem divulgados em outras formatações, configurados como revisões e materiais educativos; 3) artigos que não contivessem seu resumo nas bases de dados selecionadas; 4) artigos em meta-análise, pelo fato de não serem fontes primárias de dados; 5) artigos com deficiência na descrição metodológica, principalmente no que se refere a objetivo, métodos, resultados e conclusões. Ressalta-se a atenção observada para que os artigos não fossem incluídos duas vezes, caso estivessem indexados em mais de uma das bases de dados selecionadas.

Para o acesso e download dos textos completos e aquisição dos artigos encontrados nas bases de dados previamente selecionadas, foi utilizado o Portal de Periódicos da Capes em decorrência de convênio da Ufam, que permite o uso dessa importante ferramenta de busca de material bibliográfico.

\section{Resultados}

Foi encontrado um total de 1.405 artigos, selecionados nas bases de dados SciELO, LILACS, PubMed e MEDLINE. A Tabela 1 mostra a distribuição dos artigos encontrados e selecionados em cada base de dados.
De acordo com o ano de publicação, os artigos classificados foram os publicados entre os anos de 2001 a 2008; desses, 3 eram de 2001, 1 de 2003, 10 de 2004, 16 de 2005, 19 de 2006, 26 de 2007 e 26 de 2008. Do total de artigos, 45 foram publicados nos Estados Unidos, 31 na Inglaterra, 18 em outros países como Coreia, Japão, Alemanha, Itália, 4 artigos realizados no Brasil e 3 não continham essa informação. A Tabela 2 apresenta os artigos selecionados de acordo com os países de realização dos estudos.

Entre as regiões acometidas pela osteoartrose, o joelho foi o local mais mencionado nos estudos correspondendo a 71 dos artigos classificados, seguido pelo joelho e quadril juntos com 12 artigos, a região do quadril com 7 artigos, outras regiões como a mão, articulação temporomandibular, tornozelos corresponderam a 10 artigos e 1 artigo não mencionou a região acometida. Os principais sinais e sintomas mencionados nos artigos foram: dor e perda da função, com 44 artigos; dor, com 25 artigos; perda da função, com 14 artigos; exame radiológico, com 5 artigos; e exame radiológico e dor, com 5 artigos. 0 Quadro 1 mostra o número de artigos classificados segundo os principais sintomas.

Os estudos, apesar de apresentarem informações sobre o tipo de tratamento utilizado, em grande parte não mencionavam quantas sessões, volume ou intensidade que os exercícios foram realizados. Os artigos que mencionaram fisioterapia como forma de tratamento, estes na maioria das vezes não indicaram especificações sobre os procedimentos realizados. 0 Quadro 2 contém informações dos estudos selecionados por essa revisão sistemática, destacando os autores e ano da publicação, a região acometida, os exercícios utilizados mais mencionados nos artigos classificados e os principais resultados obtidos pelo estudo. Os artigos que constituíram o referido quadro foram aqueles que apresentaram informações mais completas, principalmente quanto aos procedimentos no uso dos tratamentos aplicados.

Tabela 1 - Quantidade total de estudos encontrados e selecionados segundo cada base de dados consultada

\begin{tabular}{ccc}
\hline Base de dados & Artigos encontrados & Totais de artigos selecionados \\
\hline MEDLINE & 505 & 54 \\
PubMed & 857 & 43 \\
LILACS & 27 & 3
\end{tabular}


Tabela 1 - Quantidade total de estudos encontrados e selecionados segundo cada base de dados consultada

(Conclusão)

\begin{tabular}{ccc}
\hline Base de dados & Artigos encontrados & Totais de artigos selecionados \\
\hline SciELO & 16 & 1 \\
Total & 1.405 & 101 \\
\hline
\end{tabular}

Fonte: Dados da pesquisa.

Tabela 2 - Quantidade de artigos selecionados segundo 0 país onde foram realizados os estudos

\begin{tabular}{lc}
\hline País de publicação & Artigos selecionados \\
\hline Estados Unidos & 45 \\
Inglaterra & 31 \\
Outros países & 18 \\
Brasil & 4 \\
Não consta & 3 \\
\hline Total & $\mathbf{1 0 1}$ \\
\hline
\end{tabular}

Fonte: Dados da pesquisa.
Quadro 1 - Quantidade de artigos selecionados segundo os principais sintomas mencionados

\begin{tabular}{lc}
\hline Principais sintomas & Número de artigos \\
\hline Dor e perda e da função física* & 44 \\
Dor & 25 \\
Perda da função física & 14 \\
Exame Radiológico & 05 \\
Exame radiológico e dor* & 05 \\
\hline
\end{tabular}

Legenda: * $=$ artigos que mencionaram os dois sintomas associados. Fonte: Dados da pesquisa.

Quadro 2 - Principais estudos selecionados de acordo com autor/ano de publicação, região acometida, tratamento utilizado e resultados

(Continua)

\begin{tabular}{llll}
\hline Autor & Região acometida & Tratamento utilizado & Resultados do estudo \\
\hline Milagres et al. (7) & Joelho & Fisioterapia & Melhora no quadro álgico e incapacitante \\
Silva et al. (8) & Joelho & Fisioterapia & $\begin{array}{l}\text { Melhora da dor, qualidade funcional e } \\
\text { flexibilidade } \\
\text { Bennell (9) }\end{array}$ \\
Van Baar et al. (10) & Joelho & Fisioterapia & Melhora da dor e da incapacidade \\
Facci et al.(11) & Joelho e/ou o quadril & Fisioterapia & Redução da dor \\
Jan et al. (12) & Joelho & Fisioterapia & $\begin{array}{l}\text { Melhora da amplitude de movimento de } \\
\text { flexão e extensão de joelho }\end{array}$ \\
Doi et al. (13) & Treino de força muscular & $\begin{array}{l}\text { Melhora da dor e da função do tempo de } \\
\text { caminhada }\end{array}$ \\
Rogers e Wilder (14) & Moelho & Treino de força muscular & Melhora da sintomatologia da osteoartrite \\
Iwamoto et al. (15) & Joelho & Treino de força muscular & Ganho de força muscular e redução da dor \\
Yurtkuran et al. (16) & Joelho & Treino de força muscular & Melhora sintomatológica da osteoartrite \\
Cochrane et al. (17) & Joelho e/ou quadril & Exercícios aquáticos & $\begin{array}{l}\text { Melhora dos sintomas clínicos e da } \\
\text { qualidade de vida }\end{array}$ \\
Deyle et al. (18) & Joelho & Caminhada & Melhora dos sinais clínicos
\end{tabular}


Quadro 2 - Principais estudos selecionados de acordo com autor/ano de publicação, região acometida, tratamento utilizado e resultados

(Conclusão)

\begin{tabular}{|c|c|c|c|}
\hline Autor & Região acometida & Tratamento utilizado & Resultados do estudo \\
\hline Focht et al. (19) & Joelho & Caminhada & Melhora na mobilidade e diminuição da dor \\
\hline Martin et al. (20) & Joelho & Caminhada & Melhora da função física e da dor \\
\hline Sato et al. (21) & Quadril & Caminhada & $\begin{array}{l}\text { Melhora da função, independência de } \\
\text { analgésico }\end{array}$ \\
\hline Eyigor et al. (22) & Joelho & Exercícios isocinéticos & Redução da dor \\
\hline Huang et al. (23) & Joelho & Exercícios isocinéticos & Melhora da função e diminuição da dor \\
\hline Cetin et al. (24) & Joelho & Exercícios isocinéticos & Melhora da função e diminuição da dor \\
\hline De Jong et al. (25) & Joelho e quadril & $\begin{array}{l}\text { Exercício e educação em } \\
\text { saúde }\end{array}$ & Redução da dor \\
\hline Coleman et al. (26) & Joelho & $\begin{array}{l}\text { Exercício e educação em } \\
\text { saúde }\end{array}$ & $\begin{array}{l}\text { Redução a dor, melhora da saúde mental e } \\
\text { da função física }\end{array}$ \\
\hline
\end{tabular}

Fonte: Dados da pesquisa.

\section{Discussão}

A revisão sistemática sobre o uso do exercício em indivíduos com osteoartrose revelou o uso deste como frequente modalidade de tratamento e o resultado parece ser bastante eficaz, já que todos os artigos classificados relataram, em seus resultados, a melhora dos sintomas clínicos referentes à dor, à função e à mobilidade. Isso possivelmente refletiu de forma direta na qualidade de vida dos indivíduos acometidos por essa doença (7-26).

Os estudos realizados no exterior apresentaram-se em maior número como mostra a Tabela 1, com o grande número de estudos nas bases de dados internacionais e o grande número de estudos realizados em países como Estados Unidos e Inglaterra, além de países como Japão, Coreia, Alemanha, como mostra a Tabela 2. Esses artigos também utilizaram amostras maiores e a média de idade era de 50 anos ou mais. A maioria dos artigos não mencionava o sexo dos indivíduos estudados e os que mencionaram utilizaram na maior parte das vezes indivíduos do sexo feminino em seus estudos (7-26).

Dentre as causas mencionadas nos estudos estão aquelas com etiologia de ocorrências traumáticas, metabólicas, inflamatórias, alterações endócrinas e defeitos congênitos. Quanto ao número de articulações envolvidas, a classificação pode ser monoarticular, oligoarticular e poliarticular, sendo esta última generalizada. Os locais de maior incidência mencionados com mais frequência referente a articulação envolvida foram o joelho, o quadril, as mãos e a coluna vertebral (27). As mulheres, contudo, tendem a apresentar a doença poliarticular mais grave, que frequentemente afeta as mãos e os joelhos. A idade, a genética e a presença de outras doenças articulares afetam as estruturas biomecânicas das articulações e influenciam a localização e a severidade do desenvolvimento da osteoartrose em uma articulação (28).

Os sintomas variam em função da gravidade das lesões (28). À medida que o processo se agrava, a dor surge aos pequenos esforços e até mesmo em repouso, sendo característica queixa ao se levantar de uma cadeira, com melhora após alguns passos (27). 0 exame físico é muito importante, pois permite que o profissional de saúde detecte o aumento de volume da articulação, a atrofia do quadríceps, além de observar dor à palpação das interlinhas articulares e à mobilização da patelar, que pode estar parcialmente ou totalmente limitada, apresentando crepitação perceptível à palpação quando realizado movimento de flexoextensão do joelho $(27,28)$. Calor e derrame articular também são característicos quando há componente inflamatório (28). A limitação da extensão do joelho e a bilateralidade do processo são causas significativas de incapacidade $(27,28)$. Como mostra o Quadro 1 , a dor associada à perda da função física apresenta-se como principal sintoma no indivíduo com osteoartrose. Os 
estudos mostram em seus resultados a considerável redução da dor e a melhora da função, assim como nos demais sintomas quando os exercícios físicos eram utilizados em um grupo comparando a outro grupo que não utilizou esse procedimento $(12,29-31)$.

Entre os tratamentos mais utilizados nos artigos classificados está o uso da fisioterapia, como apresentado no Quadro 2. Esses artigos mencionaram o uso dessa modalidade associada com o uso de medicamentos, aplicação de gelo ou calor e acupuntura (32-34). Os exercícios isométricos foram utilizados como o objetivo de aliviar a dor bem como prevenir a atrofia muscular devido à imobilidade. Para desenvolver a estabilidade articular e postural, os exercícios isotônicos foram os mais utilizados (22-24).

As outras modalidades mencionadas nos artigos verificados incluem: exercícios de força, utilizados para melhorar a estabilidade articular; exercícios de flexibilidade, que promovem o movimento da articulação de maneira suave e confortável a partir da amplitude de movimento irrestrita e sem causar dor; os alongamentos, que proporcionam o aumento da mobilidade dos tecidos moles e também melhoram a amplitude de movimento; exercício sensoriomotor; treino de habilidades; caminhadas, e educação dos pacientes em saúde acerca de sua conduta sobre a prática de exercícios e informações sobre estilo de vida saudável, além de informações sobre a doença (35-38). Em alguns estudos, os exercícios eram utilizados em associação a outras modalidades de tratamento como a crioterapia, a mobilização, ondas curtas, a estimulação elétrica nervosa transcutânea (TENS), ultrassom e a utilização combinada de vários exercícios $(8,24,39)$.

No treino de força muscular, foram utilizados exercício de baixa e alta resistência, exercícios isocinéticos que proporcionam um melhor desempenho muscular ao grupamento da articulação exercitada $(36,40)$.

0 joelho foi a região acometida mais mencionada nos artigos classificados, seguida de joelho e quadril juntos e, em algum caso, de quadril (Quadro 1), poucas vezes foi mencionada a articulação temporomandiular e da mão $(14,41)$.

Além das intervenções, os artigos descrevem suas avaliações e entre os instrumentos mais empregados estavam o Western Ontario and McMaster Universities Osteoarthritis Index (WOMAC), Medical Outcomes Study Short Form 36 Questionnaire (SF36), a Escala Visual Analógica de Dor, o Teste de Caminhada de 6-minutos, além do uso de goniômetro e dinamômetro isocinético. Estes instrumentos foram utilizados para avaliar entre outros pontos o nível de dor, a função física, a amplitude de movimento da articulação, o estado físico e mental, a qualidade de vida, os efeitos do tratamento utilizado, permitindo, assim, a comparação entre os grupos dos sujeitos estudados $(16,22,26)$.

\section{Considerações finais}

A partir da verificação dos artigos selecionados e avaliados, pôde-se averiguar que o exercício físico é empregado como método eficaz de intervenção terapêutica no tratamento da osteoartrose, na qual suas principais ações são a redução da dor e o aumento da mobilidade. A osteoartrose é uma doença articular degenerativa que pode acometer regiões do corpo que suportam grandes quantidades de peso e são muito utilizadas, o joelho é uma dessas regiões e foi comumente mencionado nos artigos dessa revisão.

A escassez de estudos no Brasil demonstra a necessidade de realizar a aplicação dessas atividades com embasamento científico. Assim sendo, são necessários mais estudos nesta área para que o emprego correto dessas modalidades de tratamento seja compreendido e reproduzido, promovendo um método baseado na evidência.

\section{Referências}

1. Coimbra IB, Pastor EH, Greve JMDA, Puccinelli MLC, Fuller R, Cavalcanti FS, et al. Consenso Brasileiro para o tratamento de Osteoartrite (Osteoartrose). Rev Bras Reumatol. 2002;42(6):371-4.

2. Rejaili WA, Chueire AG, Cordeiro JA, Petean FC, Filho GC. Avaliação do uso do Hylano GF-20 no pós-operatório de artroscopia de joelho por artrose. Acta Ortop Bras. 2005;13(1):20-3. doi:10.1590/ S1413-78522005000100005.

3. Rezende UM, Hernandez AJ, Camanho GL, Amatuzi MM. Cartilagem articular e osteoartrose. Acta Ortop Bras. 2000;8:100-4. doi:10.1590/S141378522000000200005 .

4. Camanho GL. Tratamento da osteoartrose do joelho. Rev Bras Ortop. 2001;36(5):135-40. 
5. Vasconcelos KSS, Dias JMD, Dias RC. Relação entre intensidade de dor e capacidade funcional em indivíduos obesos com osteoartrite de joelho. Rev Bras Fisioter. 2006;10(2):213-8. doi:10.1590/ S1413-35552006000200012.

6. Ricci NA, Coimbra IB. Exercício físico como tratamento na osteoartrite de quadril: uma revisão de ensaios clínicos aleatórios controlados. Rev Bras Reumatol. 2006;46(4):273-80. doi:10.1590/ S0482-50042006000400007.

7. Milagres AS, Souza IM, Pereira JOC, Paz RD, Abreu FMC. Benefícios de um programa de fortalecimento excêntrico do quadríceps no tratamento da osteoartrite de joelho. Rev Fisioter Bras. 2006;7(1):73-8.

8. Silva ALP, Imoto DM, Croci AT. Estudo comparativo entre a aplicação de crioterapia, cinesioterapia e ondas curtas no tratamento da osteoartrite de joelho. Acta Ortop Bras. 2007;15(4):204-9. doi:10.1590/ S1413-78522007000400006.

9. Bennell KL. The effects of hip muscle strengthening on knee load, pain, and function in people with knee osteoarthritis: a protocol for a randomised, single-blind controlled trial. BMC Musculoskelet Disord. 2007;8:121. doi:10.1186/1471-2474-8-121.

10. Van Baar ME, Dekker J, Oostendorp RA, Bijl D, Voorn TB, Bijlsma JW. Effectiveness of exercise in patients with osteoarthritis of hip or knee: nine months' follow up. Ann Rheum Dis. 2001;60(12):1123-30. doi:10.1136/ard.60.12.1123.

11. Facci LM, Marquetti R, Coelho KC. Fisioterapia aquática no tratamento da osteoartrite de joelho: série de casos. Fisioter Mov. 2007;20(1):17-27.

12. Jan M-H, Lin J-J, Liau J-J, Lin Y-F, Lin D-H. Investigation of clinical effects of high- and low-resistance training for patients with knee osteoarthritis: a randomized controlled trial. Phys Ther. 2008;88(4):427-36. doi:10.2522/ptj.20060300.

13. Doi T, Akai M, Fujino K, Iwaya T, Kurosawa H, Hayashi $\mathrm{K}$, et al. Effect of home exercise of quadriceps on knee osteoarthritis compared with nonsteroidal antiinflammatory drugs: a randomized controlled trial. Am J Phys Med Rehabil. 2008;87(4):258-69. doi:10.1097/ PHM.0b013e318168c02d.
14. Rogers MW, Wilder FV. The effects of strength training among persons with hand osteoarthritis: a two-year follow-up study. J Hand Ther. 2007;20(3):244-50. doi:10.1197/j.jht.2007.04.005.

15. Iwamoto J, Takeda T, Sato Y. Effect of muscle strengthening exercises on the muscle strength in patients with osteoarthritis of the knee. Knee. 2007;14(3):224-30. doi:10.1016/j.knee.2007.03.002.

16. Yurtkuran M, Yurtkuran M, Alp A, Nasircilar A, Bingol U, Altan L, et al. Balneotherapy and tap water therapy in the treatment of knee osteoarthritis. Rheumatol Int. 2006;27(1):19-27. doi:10.1007/ s00296-006-0158-8.

17. Cochrane T, Davery RC, Matthes ES. Randomised controlled trial of the cost-effectiveness of water-based therapy for lower limb osteoarthritis. Health Technol Assess. 2005;9(31):1-114.

18. Deyle GD, Allison SC, Matekel RL, Ryder MG, Stang JM, Gohdes DD, et al. Physical therapy treatment effectiveness for osteoarthritis of the knee: a randomized comparison of supervised clinical exercise and manual therapy procedures versus a home exercise program. Phys Ther. 2005;85(12):1301-7. PMid:16305269.

19. Focht BC, Rejeski WJ, Ambrosius WT, Katula JA, Messier SP. Exercise, self-efficacy, and mobility performance in overweight and obese older adults with knee osteoarthritis. Arthritis Rheum. 2005;53(5):659-65. doi:10.1002/art.21466.

20. Martin K, Fontaine KR, Nicklas BJ, Dennis KE, Goldberg AP, Hochberg MC. Weight loss and exercise walking reduce pain and improve physical functioning in overweight postmenopausal women with knee osteoarthritis. J Clin Rheumatol. 2001;7(4):219-23. doi:10.1097/00124743-200108000-00006.

21. Sato T, Yamaji T, Inose H, Sekino Y, Uchida S, Usuda $\mathrm{S}$, et al. Effect of a modified S-form hip brace, WISH type, for patients with painful osteoarthritis of the hip: a role in daily walking as a hip muscle exercise. Rheumatol Int. 2008;28(5):419-28. doi:10.1007/ s00296-007-0455-x.

22. Eyigor S, Hepguler S, Capaci KA. Comparison of muscle training methods in patients with knee osteoarthritis. Clin Rheumatol. 2005;23(2):109-15. doi:10.1007/ s10067-003-0836-9. 
23. Huang MH, Yang RC, Lee CL, Chen TW, Wang MC. Use of ultrasound to increase effectiveness of isokinetic exercise for knee osteoarthritis. Arch Phys Med Rehabil. 2005;86(8):1545-51. doi:10.1016/j. apmr.2005.02.007.

24. Cetin N, Aytar A, Atalay A, Akman MN. Comparing hot pack, short-wave diathermy, ultrasound, and TENS on isokinetic strength, pain, and functional status of women with osteoarthritic knees: a single-blind, randomized, controlled trial. Am J Phys Med Rehabil. 2008;87(6):443-51. doi:10.1097/ PHM.0b013e318174e467.

25. De Jong ORW, Hopman-Rock M, Tak ECMP, Klazinga NS. An implementation study of two evidence-based exercise and health education programmes for older adults with osteoarthritis of the knee and hip. Health Educ Res. 2004;19(3):316-25. doi:10.1093/her/ cyg028.

26. Coleman S, Briffa K, Conroy H, Prince R, Carrol G, McQuade J. Shortand medium-term effects of an education self-management program for individuals with osteoarthritis of the knee, designed and delivered by health professionals: a quality assurance study. BMC Musculoskelet Disord. 2008;8(9):117. doi:10.1186/14712474-9-117. doi:10.1186/1471-2474-9-117.

27. Hebert S, Xavier R. Ortopedia e traumatologia: princípios e prática. 3. ed. Porto alegre: Artmed; 2003.

28. Albuquerque RP, Giordano V, Sturm L, Amaral NP. Análise da reprodutibilidade de três classificações para a osteoartrose do joelho. Rev Bras Ortop. 2008;43(8):32935. doi:10.1590/S0102-36162008000800003.

29. Murphy SL, Strasburg DM, Lyden AK, Smith DM, Koliba JF, Dadabhoy DP, et al. Effects of activity strategy training on pain and physical activity in older adults with knee or hip osteoarthritis: a pilot study. Arthritis Rheum. 2008;59(10):1480-7. doi:10.1002/art.24105.

30. Oida Y, Morozumi K, Nakamura N, Kitabatake Y, Shiozawa S, Sato S, et al. Effectiveness of a community health service program using exercise intervention for elderly people with osteoarthritis of the knees: a randomized controlled trial. Nippon Koshu Eisei Zasshi. 2008;55(4):228-37. PMid:18536337.
31. Shen CL, James CR, Chyu MC, Bixby WR, Brismée JM, Zumwalt MA, et al. Effects of Tai Chi on gait kinematics, physical function, and pain in elderly with knee osteoarthritis - a pilot study. Am J Chin Med. 2008;36(2):21932. doi:10.1142/S0192415X08005734.

32. Domaille M, Mascarenhas R, Dayal N, Kirwan J. Evaluation of the Bristol Royal Infirmary physiotherapy programme for the management of patients with osteoarthritis of the knee. Musculoskeletal Care. 2006;4(2):78-87. doi:10.1002/msc.80.

33. Williamson L, Wyatt MR, Yein K, Melton JT. Severe knee osteoarthritis: a randomized controlled trial of acupuncture, physiotherapy (supervised exercise) and standard management for patients awaiting knee replacement. Rheumatology (Oxford). 2007;46(9):14459. doi:10.1093/rheumatology/kem119.

34. Tsang RC, Tsang PL, Ko CY, Kong BC, Lee WY, Yip HT. Effects of acupuncture and sham acupuncture in addition to physiotherapy in patients undergoing bilateral total knee arthroplasty - a randomized controlled trial. Clin Rehabil. 2007;21(8):719-28. doi:10.1177/0269215507077362.

35. Crossley KM, Vicenzino B, Pandy MG, Schache AG, Hinman RS. Targeted physiotherapy for patellofemoral joint osteoarthritis: a protocol for a randomised, single-blind controlled trial. BMC Musculoskelet Disord. 2008;9:122. doi:10.1186/1471-2474-9-122.

36. Kisner C, Colby LA. Exercícios terapêuticos. 4. ed. Barueri: Manole; 2005.

37. De Jong OR, Hopman-Rock M, Tak EC, Klazinga NS. An implementation study of two evidence-based exercise and health education programmes for older adults with osteoarthritis of the knee and hip. Health Educ Res. 2004;19(3):316-25. doi:10.1093/her/cyg028.

38. Cheon EY. The effects of a self-management program on physical function and quality of life of patients with knee osteoarthritis. Taehan Kanho Hakhoe Chi. 2005;35(3):514-25. PMid:16027503.

39. Comim AT, Perea DC. Análise da eficácia do uso do ultra-som e alongamento (inibição ativa) na avaliação do escore de dor em pacientes portadores de artrose de joelho. Reabilitar. 2004;6(25):14-9. 
40. Sato T, Yamaji T, Inose H, Sekino Y, Uchida S, Usuda $\mathrm{S}$, et al. Effect of a modified S-form hip brace, WISH type, for patients with painful osteoarthritis of the hip: a role in daily walking as a hip muscle exercise. Rheumatol Int. 2008;28(5):419-28. doi:10.1007/ s00296-007-0455-x.

41. Nicolakis P, Erdogmus CB, Kollmitzer J, Kerschan-Schindl K, Sengstbratl M, Nuhr M, et al. Long-term outcome after treatment of temporomandibular joint osteoarthritis with exercise and manual therapy. Cranio. 2002;20(1):23-7. PMid:11831340.

Recebido: 16/01/2012

Received: 01/16/2012

Aprovado: 08/08/2012

Approved: 08/08/2012 